\title{
Leading Electroweak Corrections to the Neutral Higgs Boson Production at the Fermilab Tevatron
}

\author{
Qing Hong Cao, Chong Sheng Li \\ Department of Physics, Peking University, Beijing 100871, P. R. China \\ Shou Hua Zhu \\ CCAST (World Laboratory), P. O. Box 8730, Beijing 100080, P. R. China \\ Institute of Theoretical Physics, Academia Sinica, P. O. Box 2735, Beijing 100080, P. R. \\ China \\ ABSTRACT

\begin{abstract}
We calculate the leading electroweak corrections to the light neutral Higgs boson production via $q \bar{q}^{\prime} \rightarrow W H$ at the Fermilab Tevatron in both the standard model and the minimal supersymmetric model, which arise from the top-quark and Higgs boson loop diagrams. We found that the leading electroweak corrections can exceed the QCD corrections for favorable values of the parameters in the MSSM, but such corrections are only about $-1 \% \sim-2 \%$ in the SM, which are much smaller than the QCD corrections. For the mass region of $90<m_{h_{0}}<120 \mathrm{GeV}$, the leading electroweak corrections can reach $-10 \%$ for large $\tan \beta$, and these corrections may be observable at a high luminosity Tevatron; at the least, new constraints on the
\end{abstract} \\ $\tan \beta$ can be established.
}

PACS number: 14.80.Bn, 14.80.Cp, 13.85.QK, 12.60.Jv 


\section{INTRODUCTION}

One of the most important physics goals for future high energy physics is the discovery of the Higgs boson. Recent direct search in the LEP2 experiments of running at $\sqrt{s}=183$ $\mathrm{GeV}$ via the $e^{+} e^{-} \rightarrow Z^{*} H$ yields a lower bound of $\sim 89.9 \mathrm{GeV}$ on the Higgs mass [1]. Next year's running at $192 \mathrm{GeV}$ will explore up to a Higgs boson mass of about $96 \mathrm{GeV}$ [2]. After LEP2 the search for the Higgs particles will be continued at the CERN Large Hadron Collider (LHC) for Higgs boson masses up to the theoretical upper limit. Before the LHC comes into operation it is worth considering whether the Higgs boson can be discovered from the existing hadron collider, the Tevatron. Much study has been made in the detection of a Higgs boson at the Tevatron [3]. In Ref. [2], it was pointed out that if the Higgs boson is discovered at LEP2, it should be observed at the Tevatron's Run II with CM energy $\sqrt{s}=2$ $\mathrm{TeV}$ and an integrated luminosity $\sim 10 \mathrm{fb}^{-1}$, through the production subprocess $q \bar{q}^{\prime} \rightarrow W H$, followed by $W \rightarrow \ell \bar{\nu}$ and $H \rightarrow b \bar{b}$, and if the Higgs boson lies beyond the reach of LEP2, $m_{H} \geq(95-100) \mathrm{GeV}$, then a $5-\sigma$ discovery will be possible in the above production subprocess in a future Run III with an integrated luminosity $30 \mathrm{fb}^{-1}$ for masses up to $m_{H} \approx 125$ $\mathrm{GeV}$. Since the expected number of events is small, it is important to calculate the cross section as accurately as possible. In Ref. [4 the $O\left(\alpha_{s}\right)$ QCD correction to the total cross section to this process have been calculated, and the QCD correction were found to be about $12 \%$ in the $\overline{M S}$ scheme at the Fermilab Tevatron and the LHC in the Standard Model(SM). In general, the SM electroweak corrections are small comparing with the QCD correction. Beyond the SM, the electroweak corrections might be enhanced, since more Higgs bosons and the top quark with stronger couplings are involved in the loop diagrams; for example, in the

minimal supersymmtric model(MSSM) [5], which predict that the lightest Higgs boson $h_{0}$ be less than $140 \mathrm{GeV}$ [6]. Therefore, it is worthwhile to calculate the electroweak corrections to the light Higgs boson production via $q \bar{q}^{\prime} \rightarrow W h_{0}$. In a previous paper [7] we calculated the $O\left(\alpha_{e w} m_{t}^{2} / m_{W}^{2}\right)$ corrections arising from the top quark loops to this process in both the SM and the MSSM and found that in contrast to the QCD corrections which increases the tree-level cross sections, such corrections reduce the cross sections by about $1 \% \sim 2 \%$ in the $\mathrm{SM}$, and $1 \% \sim 4 \%$ in the MSSM. However, in addition to these top quark loops corrections, the Higgs boson loops corrections should also be taken into account, which are of order 
$Q\left(\alpha_{\text {ew }} m_{H}^{2} / m_{W}^{2}\right)$, especially in the MSSM, such corrections may be comparable to the top quark loops corrections, and even exceed them for large $\tan \beta$. In this paper, we consider the leading electroweak radiative corrections to the Higgs boson production at the Fermilab Tevatron in both the SM and the MSSM. These corrections arise from the virtual effects of the third family (top and bottom) of quark, neutral and charged Higgs bosons, and neutral and charged Goldstone bosons. We present the corrections to production cross section versus Higgs boson mass changing in the region of $60 \mathrm{GeV} \leq m_{h_{0}} \leq 130 \mathrm{GeV}$ for different values

of $\tan \beta$, and compare with the results of the top quark loops in the SM and the MSSM, and also compare with the QCD corrections.

\section{CACULATIONS}

The leading electroweak corrections to the process $q\left(p_{1}\right) \bar{q}^{\prime}\left(p_{2}\right) \rightarrow W\left(k_{1}\right) h_{0}\left(k_{2}\right)$ arise from the Feynman diagrams shown in Fig 1-4. We perform the calculation in the 't HooftFeynman gauge and use dimensional regularization to all the ultraviolet divergence in the virtual loop corrections utilizing the on-mass-shell renormalization [8], in which the finestructure constant $\alpha$ and the physical masses are chosen to be the renormalized parameters, and the finite parts of the countertems are fixed by the renormalization conditions. As far as the parameters $\beta$ and $\alpha$, for the MSSM we are considering, they have to be renormalized, too. In the MSSM they are not independent. Nevertheless, we follow the approach of Mendez and Pomarol [9] in which they consider them as independent renormalized parameters and fixed the corresponding renormalization constant by a renormalization condition that the on-mass-shell $H^{+} \bar{\ell} \nu_{\ell}$ and $h_{0} \bar{\ell} \ell$ couplings keep the forms of Eq.(3) of Ref. [9] to all order of perturbation theory.

We define the Mandelstam variables as

$$
\begin{aligned}
& \hat{s}=\left(p_{1}+p_{2}\right)^{2}=\left(k_{1}+k_{2}\right)^{2} \\
& \hat{t}=\left(p_{1}-k_{1}\right)^{2}=\left(p_{2}-k_{2}\right)^{2} \\
& \hat{u}=\left(p_{1}-k_{2}\right)^{2}=\left(p_{2}-k_{1}\right)^{2} .
\end{aligned}
$$

The relevant renormalization constants are defined as 


$$
\begin{gathered}
m_{W 0}^{2}=m_{W}^{2}+\delta m_{W}^{2}, \quad m_{Z 0}^{2}=m_{Z}^{2}+\delta m_{Z}^{2}, \\
\tan \beta_{0}=\left(1+\delta Z_{\beta}\right) \tan \beta, \quad \sin \alpha_{0}=\left(1+\delta Z_{\alpha}\right) \sin \alpha, \\
W_{0}^{ \pm \mu}=Z_{W}^{1 / 2} W^{ \pm \mu}+i Z_{H^{ \pm} W}^{1 / 2} \partial^{\mu} H^{ \pm}, \quad H_{0}^{ \pm}=\left(1+\delta Z_{H^{ \pm}}\right)^{1 / 2} H^{ \pm}, \\
h_{0}=\left(1+\delta h_{0}\right)^{1 / 2} h+Z_{h_{0} H}^{1 / 2} H, \quad H_{0}=\left(1+\delta Z_{H}\right)^{1 / 2} H+Z_{H h_{0}}^{1 / 2} h
\end{gathered}
$$

Taking into account the leading electroweak corrections, the renormalized amplitude for $q \bar{q}^{\prime} \rightarrow W h_{0}$ can be written as

$$
M_{\text {ren }}=M_{0}+\delta M^{\text {self }}+\delta M^{\text {vertex }}
$$

where $M_{0}$ is the amplitude at the tree level, $\delta M^{\text {self }}$ and $\delta M^{\text {vertex }}$ represent the corrections arising from the self-energy and vertex diagrams, respectively. $M_{0}$ is given by

$$
M_{0}=\frac{e^{2} m_{W} \sin (\beta-\alpha)}{\sqrt{2}\left(m_{W}^{2}-\hat{s}\right) \sin \theta_{w}^{2}} \bar{d}\left(p_{2}\right) \notin P_{L} u\left(p_{1}\right),
$$

where $P_{L, R} \equiv\left(1 \mp \gamma_{5}\right) / 2$. $\delta M^{\text {self }}$ is given by

$$
\delta M^{\text {self }}=\frac{\delta m_{W}^{2}+\left(m_{W}^{2}-\hat{s}\right) \delta Z_{W}}{\hat{s}-m_{W}^{2}} M_{0}+\delta M_{t}^{\text {self }}+\delta M_{H}^{\text {self }}
$$

$\delta M_{t}^{\text {self }}$ represents the correction arising from the top-quark self-energy diagrams to the order $O\left(\alpha_{\text {ew }} m_{t}^{2} / m_{W}^{2}\right), \delta M_{H}^{\text {self }}$ represents the corrections arising from the Higgs boson self-energy diagrams to the order $O\left(\alpha_{e w} m_{H}^{2} / m_{W}^{2}\right) . H=M_{H}^{ \pm}, h, M_{H}^{0}, A$. The renormalization constant $\delta m_{W}^{2}$ and $\delta Z_{W}$ are presented in the appendix-A. $\delta M_{t}^{\text {self }}$ and $\delta M_{H}^{\text {self }}$ are presented in the Appendix-B.

$\delta M^{\text {vertex }}$ is given by

$$
\begin{aligned}
\delta M^{\text {vertex }} & =M_{0}\left[\frac{1}{2} \delta Z_{h_{0}}+\frac{\delta m_{W}^{2}-\delta m_{Z}^{2}}{2\left(m_{Z}^{2}-m_{W}^{2}\right)}+\frac{\delta m_{Z}^{2}}{m_{Z}^{2}}+\frac{\delta m_{W}^{2}}{m_{W}^{2}}+\frac{\delta e}{e}\right. \\
& \left.+\cot (\beta-\alpha)\left(Z_{H h_{0}}^{1 / 2}+\sin \beta \cos \beta \delta Z_{\beta}-\tan \alpha \delta Z_{\alpha}\right)\right] \\
& +f_{1}^{\text {vertex }} \bar{d}\left(p_{2}\right) \notin P_{L} u\left(p_{1}\right) \\
& +f_{2}^{\text {vertex }} \bar{d}\left(p_{2}\right) p_{1} P_{L} u\left(p_{1}\right) \epsilon \cdot p_{1} \\
& +f_{3}^{\text {vertex }} \bar{d}\left(p_{2}\right) p_{1} P_{L} u\left(p_{1}\right) \epsilon \cdot p_{2},
\end{aligned}
$$


where

$$
\begin{gathered}
\delta Z_{\beta}=\frac{\delta m_{Z}^{2}-\delta m_{W}^{2}}{2\left(m_{Z}^{2}-m_{W}^{2}\right)}-\frac{\delta m_{Z}^{2}}{2 m_{Z}^{2}}+\frac{\delta m_{W}^{2}}{2 m_{W}^{2}}-\frac{1}{2} \delta Z_{H^{ \pm}}-\frac{m_{W}}{\tan \beta} Z_{W H^{ \pm}}^{1 / 2}, \\
\delta Z_{\alpha}=-\sin ^{2} \beta \delta Z_{\beta}+\frac{\delta m_{Z}^{2}-\delta m_{W}^{2}}{2\left(m_{Z}^{2}-m_{W}^{2}\right)}-\frac{\delta m_{Z}^{2}}{2 m_{Z}^{2}}+\frac{\delta m_{W}^{2}}{2 m_{W}^{2}} \\
-\frac{1}{2} \delta Z_{h_{0}}+\frac{\cos \alpha}{\sin \alpha} Z_{H h_{0}}^{1 / 2},
\end{gathered}
$$

Note that $\delta e / e$ appearing in Eq. (9) contains no $O\left(\alpha m_{t}^{2} / m_{W}^{2}\right)$ or $O\left(\alpha m_{H}^{2} / m_{W}^{2}\right)$ terms and need not be considered in our calculations. Although the renormalization constant $Z_{H h_{0}}^{1 / 2}$ appears in $\delta Z_{\alpha}$ and the vertex counterterm, they cancel each other with accuracy and give no contribution to our calculation. Others renormalization constants are listed in the Appendix-A and the vertex factors $f_{1,2,3}^{v e r t e x}$ are given in the Appendix-C.

The corresponding amplitude squared for the process $q \bar{q}^{\prime} \rightarrow W h_{0}$ can be written as

$$
\bar{\sum}\left|M_{r e n}\right|^{2}=\bar{\sum}\left|M_{0}\right|^{2}+2 R e \bar{\sum}\left(\delta M^{\text {self }}+\delta M^{\text {vertex }}\right) M_{0}^{\dagger},
$$

where the bar over the summation recalls average over initial partons spins. The cross section of process $q \bar{q}^{\prime} \rightarrow W h_{0}$ is

$$
\hat{\sigma}=\int_{\hat{t}_{\text {min }}}^{\hat{t}_{\text {max }}} \frac{1}{16 \pi \hat{s}^{2}} \sum_{\text {spins }}^{-}|M|^{2} d \hat{t}
$$

with

$$
\begin{gathered}
\hat{t}_{\text {min }}=\frac{m_{h_{0}}^{2}+m_{W}^{2}-\hat{s}}{2}-\sqrt{\left(\hat{s}-\left(m_{h_{0}}+m_{W}\right)^{2}\right)\left(\hat{s}-\left(m_{h_{0}}-m_{W}\right)^{2}\right) / 2} \\
\hat{t}_{\text {max }}=\frac{m_{h_{0}}^{2}+m_{W}^{2}-\hat{s}}{2}+\sqrt{\left(\hat{s}-\left(m_{h_{0}}+m_{W}\right)^{2}\right)\left(\hat{s}-\left(m_{h_{0}}-m_{W}\right)^{2}\right) / 2} .
\end{gathered}
$$

The total cross section of $P \bar{P} \rightarrow q \bar{q}^{\prime} \rightarrow W h_{0}$ can be obtained by folding the $\hat{\sigma}$ with the parton luminosity

$$
\sigma(s)=\int_{\left(m_{h_{0}}+m_{W}\right) / \sqrt{s}}^{1} d z \frac{d L}{d z} \hat{\sigma}\left(q \bar{q}^{\prime} \rightarrow W h_{0} \text { at } \hat{s}=z^{2} s\right)
$$

where $\sqrt{s}$ and $\sqrt{\hat{s}}$ is the CM energy of $P \bar{P}$ and $q \bar{q}^{\prime}$, respectively, and $d L / d z$ is the parton luminosity, which is defined as

$$
\frac{d L}{d z}=2 z \int_{z^{2}}^{1} \frac{d x}{x} f_{q / P}\left(x, q^{2}\right) f_{q^{\prime} / \bar{P}}\left(z^{2} / x, q^{2}\right),
$$

where $f_{q / P}\left(x, q^{2}\right)$ and $f_{q^{\prime} / \bar{P}}\left(z^{2} / x, q^{2}\right)$ are the parton distribution function 10 . 


\section{NUMERICAL RESULTS}

In our numerical calculations, the SM parameters were taken to be $m_{W}=80.33 \mathrm{GeV}$, $m_{Z}=91.187 \mathrm{GeV}, m_{t}=176 \mathrm{GeV}, m_{b}=4.5 \mathrm{GeV}$ and $\alpha_{e w}=\frac{1}{128}$. Moreover, we use the relation [11] between the Higgs boson masses $m_{h_{0}, H, A, H^{ \pm}}$and parameters $\alpha, \beta$ at one-loop, and choose $m_{h_{0}}$ and $\tan \beta$ as two independent input parameters. As explained in Ref. [9], there is a small inconsistency in doing so since the parameters $\alpha$ and $\beta$ of Ref. [6] are not the ones defined by the conditions given by Eq.(3) of Ref. [9]. Nevertheless, this difference would only induce a higher order change [9]. We will limit the value of $\tan \beta$ to be in the range $2 \leq \tan \beta \leq 30$, which are consistent with ones required by the most popular MSSM model with scenarios motivated by current low energy data (including $\alpha_{s}, R_{b}$ and the branching ratio of $b \rightarrow s \gamma)$.

In Figs. 5 and 6 we present both the top quark loop corrections [7] and the leading electroweak radiative corrections as a function of $m_{h}$ for the different values of $\tan \beta$ using the CTEQ3L parton distributions for the tree-level cross sections $\sigma_{0}$ and the CTEQ3M parton distributions for the corrections $\delta \sigma$. From Fig. 5 one sees that the leading electroweak corrections are almost the same as the top quark loop corrections in the SM, which means that the corrections arising from the Higgs boson loops are negligibly small in the SM. And these corrections are not sensitive to the mass of the Higgs boson and amount to $1 \% \sim 2 \%$ reduction in the tree-level total cross sections. However, in the MSSM, the Higgs boson loops corrections are important, and especially for large $\tan \beta(>4)$, they can exceed the top quark loop corrections. As a result, the leading electroweak corrections are much larger than the top quark loops corrections. As shown in Fig. 6, for $\tan \beta=30$, the leading electroweak corrections decrease the cross section by $35 \%$ when $m_{h}=60 \mathrm{GeV}$, while the top quark loops corrections decrease only about $-4 \%$. However, these corrections are sensitive

to $m_{h_{0}}$. For $m_{h_{0}}$ in the range $90-120 \mathrm{GeV}$, the leading electroweak corrections and the top quark loops corrections drop to about $-10 \% \sim-2 \%$ and $-3 \% \sim-1 \%$, respectively, which indicate that the leading electroweak corrections are still obviously larger than one from the top quark loops. Only in the vicinity of $m_{h_{0}} \approx 130 \mathrm{GeV}$ for all values of $\tan \beta$ are the leading electroweak and top loop corrections about the same as one in the SM.

Comparing with the QCD corrections to this process [4], which increase the tree-level 
total cross sections by about $12 \%$, we find that in general the leading electroweak corrections partly cancel the QCD corrections, but for large $\tan \beta$, the magnitude of the former can even exceed the latter, and have to be considered in searching the light Higgs boson in the MSSM through this process at the Fermilab Tevatron.

To summarize, the leading electroweak radiative corrections, which combine the top quark and the Higgs boson loops contributions, can exceed the QCD corrections for favorable values of the parameters in the MSSM, but such corrections are only about $-1 \% \sim-2 \%$ in the SM, which are much smaller than the QCD corrections. The mass region of $90<$ $m_{h_{0}}<120 \mathrm{GeV}$ is the interesting window for searching the Higgs boson at the Tevatron, in which the leading electroweak corrections vary from $-10 \%$ to $-2 \%$ for $\tan \beta=30$, and these corrections may be observable at a high luminosity Tevatron; at the least, new constraints on the $\tan \beta$ can be established.

\section{ACKNOWLEDGMENTS}

This work was supported in part by the National Natural Science Foundation of China and a grant from the State Commission of Science and Technology of China.

\section{APPENDIX-A: THE RENORMALIZATION CONSTANTS}

$$
\begin{aligned}
\delta m_{W}^{2} & =\frac{N_{c} e^{2} m_{t}^{2}}{96 \pi^{2} \sin ^{2} \theta_{W}}\left[-2+\frac{m_{t}^{2}}{m_{W}^{2}}\left[B_{0}\left(0, m_{b}^{2}, m_{t}^{2}\right)-B_{0}\left(m_{W}^{2}, m_{b}^{2}, m_{t}^{2}\right)\right]\right. \\
& \left.+2 B_{0}\left(0, m_{b}^{2}, m_{t}^{2}\right)-B_{0}\left(m_{W}^{2}, m_{b}^{2}, m_{t}^{2}\right)-4 B_{0}\left(0, m_{t}^{2}, m_{t}^{2}\right)\right] \\
& +\frac{e^{2} \sin ^{2}(\beta-\alpha)}{192 \pi^{2} \sin ^{2} \theta_{W}}\left[-2 m_{H}^{2}-4 m_{H^{+}}{ }^{2}\right. \\
& +\frac{m_{H}^{4}}{m_{W}^{2}}\left[B_{0}\left(m_{W}^{2}, m_{H}^{2}, m_{H^{+}}^{2}\right)-B_{0}\left(0, m_{H}^{2}, m_{H^{+}}{ }^{2}\right)\right] \\
& +\frac{2 m_{H}^{2} m_{H^{+}}{ }^{2}}{m_{W}^{2}}\left[B_{0}\left(0, m_{H}^{2}, m_{H^{+}}{ }^{2}\right)-B_{0}\left(m_{W}^{2}, m_{H}^{2}, m_{H^{+}}{ }^{2}\right)\right] \\
& +\frac{m_{H^{+}}{ }^{4}}{m_{W}^{2}}\left[B_{0}\left(m_{W}^{2}, m_{H}^{2}, m_{H^{+}}{ }^{2}\right)-B_{0}\left(0, m_{H}^{2}, m_{H^{+}}{ }^{2}\right)\right] \\
& +m_{H}^{2}\left[-2 B_{0}\left(m_{W}^{2}, m_{H}^{2}, m_{H^{+}}{ }^{2}\right)-B_{0}\left(0, m_{H}^{2}, m_{H^{+}}{ }^{2}\right)\right] \\
& \left.+m_{H^{+}}{ }^{2}\left[-2 B_{0}\left(m_{W}^{2}, m_{H}^{2}, m_{H^{+}}{ }^{2}\right)+B_{0}\left(0, m_{H}^{2}, m_{H^{+}}{ }^{2}\right)-2 B_{0}\left(0, m_{H^{+}}^{2}, m_{H^{+}}{ }^{2}\right)\right]\right]
\end{aligned}
$$




$$
\begin{aligned}
& +\frac{e^{2} \cos ^{2}(\beta-\alpha)}{192 \pi^{2} \sin ^{2} \theta_{W}}\left[-2 m_{h_{0}}^{2}-4 m_{H^{+}}^{2}\right. \\
& +\frac{m_{H^{+}}{ }^{4}}{m_{W}^{2}}\left[B_{0}\left(m_{W}^{2}, m_{H^{+}}{ }^{2}, m_{h_{0}}{ }^{2}\right)-B_{0}\left(0, m_{H^{+}}{ }^{2}, m_{h_{0}}{ }^{2}\right)\right] \\
& +\frac{2 m_{h_{0}}{ }^{2} m_{H^{+}}{ }^{2}}{m_{W}^{2}}\left[B_{0}\left(0, m_{H^{+}}{ }^{2}, m_{h_{0}}{ }^{2}\right)-B_{0}\left(m_{W}^{2}, m_{H^{+}}{ }^{2}, m_{h_{0}}{ }^{2}\right)\right] \\
& +\frac{m_{h_{0}}{ }^{4}}{m_{W}^{2}}\left[B_{0}\left(m_{W}^{2}, m_{H^{+}}{ }^{2}, m_{h_{0}}{ }^{2}\right)-B_{0}\left(0, m_{H^{+}}{ }^{2}, m_{h_{0}}{ }^{2}\right)\right] \\
& +m_{H^{+}}{ }^{2}\left[-2 B_{0}\left(m_{W}{ }^{2}, m_{H^{+}}{ }^{2},{m_{h_{0}}}^{2}\right)-2 B_{0}\left(0, m_{H^{+}}{ }^{2}, m_{H^{+}}{ }^{2}\right)+B_{0}\left(0, m_{H^{+}}{ }^{2}, m_{h_{0}}{ }^{2}\right)\right] \\
& \left.+m_{h_{0}}{ }^{2}\left[-B_{0}\left(0, m_{H^{+}}{ }^{2}, m_{h_{0}}{ }^{2}\right)-2 B_{0}\left(m_{W}^{2}, m_{H^{+}}{ }^{2}, m_{h_{0}}{ }^{2}\right)\right]\right] \\
& +\frac{e^{2}}{192 \pi^{2} \sin ^{2} \theta_{W}}\left[-2 m_{H^{+}}{ }^{2}-4 m_{A}^{2}\right. \\
& +\frac{m_{A}^{4}}{m_{W}^{2}}\left[B_{0}\left(m_{W}^{2}, m_{A}^{2}, m_{H^{+}}{ }^{2}\right)-B_{0}\left(0, m_{A}^{2}, m_{H^{+}}{ }^{2}\right)\right] \\
& +\frac{2 m_{A}^{2} m_{H^{+}}^{2}}{m_{W}^{2}}\left[B_{0}\left(0, m_{A}^{2}, m_{H^{+}}^{2}\right)-B_{0}\left(m_{W}^{2}, m_{A}^{2}, m_{H^{+}}{ }^{2}\right)\right] \\
& +\frac{m_{H^{+}}^{4}}{m_{W}^{2}}\left[B_{0}\left(m_{W}^{2}, m_{A}^{2}, m_{H^{+}}{ }^{2}\right)-B_{0}\left(0, m_{A}^{2}, m_{H^{+}}{ }^{2}\right)\right] \\
& +m_{A}^{2}\left[-2 B_{0}\left(m_{W}^{2}, m_{A}^{2}, m_{h^{+}}{ }^{2}\right)-2 B_{0}\left(0, m_{A}^{2}, m_{A}^{2}\right)+B_{0}\left(0, m_{A}^{2}, m_{H^{+}}{ }^{2}\right)\right] \\
& \left.+m_{H^{+}}{ }^{2}\left[-B_{0}\left(0, m_{A}^{2}, m_{H^{+}}{ }^{2}\right)-2 B_{0}\left(m_{W}^{2}, m_{A}^{2}, m_{H^{+}}{ }^{2}\right)\right]\right] \\
& +\frac{e^{2} \cos ^{2}(\beta-\alpha)}{192 \pi^{2} \sin ^{2} \theta_{W}}\left[-4 m_{H}^{2}+\frac{m_{H}^{4}}{m_{W}^{2}}\left[B_{0}\left(m_{W}^{2}, m_{H}^{2}, m_{W}^{2}\right)-B_{0}\left(0, m_{H}^{2}, m_{W}^{2}\right)\right]\right. \\
& \left.+m_{H}^{2}\left[-4 B_{0}\left(m_{W}^{2}, m_{H}^{2}, m_{W}^{2}\right)-2 B_{0}\left(0, m_{H}^{2}, m_{H}^{2}\right)+3 B_{0}\left(0, m_{H}^{2}, m_{W}^{2}\right)\right]\right] \\
& +\frac{e^{2} \sin ^{2}(\beta-\alpha)}{192 \pi^{2} \sin ^{2} \theta_{W}}\left[-4 m_{h_{0}}{ }^{2}+\frac{m_{h_{0}}{ }^{4}}{m_{W}^{2}}\left[B_{0}\left(m_{W}^{2}, m_{h_{0}}{ }^{2}, m_{W}^{2}\right)-B_{0}\left(0, m_{h_{0}}{ }^{2}, m_{W}^{2}\right)\right]\right. \\
& \left.+m_{h_{0}}{ }^{2}\left[-4 B_{0}\left(m_{W}^{2}, m_{h_{0}}{ }^{2}, m_{W}^{2}\right)-2 B_{0}\left(0, m_{h_{0}}{ }^{2}, m_{h_{0}}{ }^{2}\right)+3 B_{0}\left(0, m_{h_{0}}{ }^{2}, m_{W}{ }^{2}\right)\right]\right] \\
& +\frac{e^{2} m_{H}^{2}}{64 \pi^{2} \sin ^{2} \theta_{W}}\left[1+B_{0}\left(0, m_{H}^{2}, m_{H}^{2}\right)\right] \\
& +\frac{e^{2} m_{H^{+}}{ }^{2}}{32 \pi^{2} \sin ^{2} \theta_{W}}\left[1+B_{0}\left(0, m_{H^{+}}{ }^{2}, m_{H^{+}}{ }^{2}\right)\right] \\
& +\frac{e^{2} m_{A}^{2}}{64 \pi^{2} \sin ^{2} \theta_{W}}\left[1+B_{0}\left(0, m_{A}^{2}, m_{A}^{2}\right)\right] \\
& +\frac{e^{2} m_{h_{0}}{ }^{2}}{64 \pi^{2} \sin ^{2} \theta_{W}}\left[1+B_{0}\left(0, m_{h_{0}}{ }^{2}, m_{h_{0}}{ }^{2}\right)\right]
\end{aligned}
$$

$$
\begin{aligned}
\delta m_{Z}^{2} & =\frac{N_{C} e^{2} m_{t}^{2}}{288 \cos ^{2} \theta_{W} \pi^{2} \sin ^{2} \theta_{W}}\left[-18 B_{0}\left(0, m_{t}^{2}, m_{t}^{2}\right)+48 \sin \left(\theta_{W}\right)^{2} B_{0}\left(0, m_{t}^{2}, m_{t}^{2}\right)\right. \\
& -64 \sin ^{4} \theta_{W} B_{0}\left(0, m_{t}^{2}, m_{t}^{2}\right)-9 B_{0}\left(m_{Z}^{2}, m_{t}^{2}, m_{t}^{2}\right)
\end{aligned}
$$




$$
\begin{aligned}
& \left.-48 \sin ^{2} \theta_{W} B_{0}\left(m_{Z}^{2}, m_{t}^{2}, m_{t}^{2}\right)+64 \sin ^{2} \theta_{W} B_{0}\left(0, m_{t}^{2}, m_{t}^{2}\right)\right] \\
& +\frac{e^{2}\left(\sin ^{2} \theta_{W}-\cos ^{2} \theta_{W}\right)^{2}}{96 \cos ^{2} \theta_{W} \pi^{2} \sin ^{2} \theta_{W}}\left[-3 m_{H^{+}}{ }^{2}-m_{H^{+}}{ }^{2} B_{0}\left(0, m_{H^{+}}{ }^{2}, m_{H^{+}}{ }^{2}\right)\right. \\
& \left.-2 m_{H^{+}}{ }^{2} B_{0}\left(m_{Z}^{2}, m_{H^{+}}{ }^{2}, m_{H^{+}}{ }^{2}\right)\right] \\
& +\frac{e^{2} \cos ^{2}(\alpha-\beta)}{192 \cos ^{2} \theta_{W} \pi^{2} \sin ^{2} \theta_{W}}\left[-2 m_{H}^{2}+\frac{m_{H}^{4}}{m_{Z}^{2}}\left[B_{0}\left(m_{Z}^{2}, m_{H}^{2}, m_{Z}^{2}\right)-B_{0}\left(0, m_{H}^{2}, m_{Z}^{2}\right)\right]\right. \\
& \left.+m_{H}^{2}\left[B_{0}\left(0, m_{H}^{2}, m_{Z}^{2}\right)-4 B_{0}\left(m_{Z}^{2}, m_{H}^{2}, m_{Z}^{2}\right)\right]\right] \\
& +\frac{e^{2} \sin ^{2}(\alpha-\beta)}{192 \cos ^{2} \theta_{W} \pi^{2} \sin ^{2} \theta_{W}}\left[-2 m_{H}^{2}-4 m_{A}^{2}\right. \\
& +\frac{\left(m_{H}^{2}-m_{A}^{2}\right)^{2}}{m_{Z}^{2}}\left[B_{0}\left(m_{Z}^{2}, m_{A}^{2}, m_{H}^{2}\right)-B_{0}\left(0, m_{A}^{2}, m_{H}^{2}\right)\right] \\
& +m_{H}^{2}\left[-B_{0}\left(0, m_{A}^{2}, m_{H}^{2}\right)-2 B_{0}\left(m_{Z}^{2}, m_{A}^{2}, m_{H}^{2}\right)\right] \\
& \left.+m_{A}^{2}\left[-2 B_{0}\left(m_{Z}^{2}, m_{A}^{2}, m_{H}^{2}\right)-2 B_{0}\left(0, m_{A}^{2}, m_{A}^{2}\right)+B_{0}\left(0, m_{A}^{2}, m_{H}^{2}\right)\right]\right] \\
& +\frac{e^{2} \sin ^{2}(\alpha-\beta)}{192 \cos ^{2} \theta_{W} \pi^{2} \sin ^{2} \theta_{W}}\left[-2 m_{h_{0}}{ }^{2}+\frac{m_{h_{0}}^{4}}{m_{Z}^{2}}\left[B_{0}\left(m_{Z}^{2}, m_{h_{0}}{ }^{2}, m_{Z}^{2}\right)-B_{0}\left(0, m_{h_{0}}{ }^{2}, m_{Z}^{2}\right)\right]\right. \\
& \left.+m_{h_{0}}{ }^{2}\left[B_{0}\left(0, m_{h_{0}}{ }^{2}, m_{Z}^{2}\right)-4 B_{0}\left(m_{z}^{2}, m_{h_{0}}{ }^{2}, m_{Z}^{2}\right)\right]\right] \\
& +\frac{e^{2} \cos ^{2}(\alpha-\beta)}{192 \cos ^{2} \theta_{W} \pi^{2} \sin ^{2} \theta_{W}}\left[-2 m_{h_{0}}{ }^{2}-4 m_{A}^{2}\right. \\
& +\frac{\left(m_{h_{0}}{ }^{2}-m_{A}^{2}\right)^{2}}{m_{Z}^{2}}\left[B_{0}\left(m_{Z}^{2}, m_{A}^{2}, m_{h_{0}}^{2}\right)-B_{0}\left(0, m_{A}^{2}, m_{h_{0}}^{2}\right)\right] \\
& +m_{h_{0}}{ }^{2}\left[-B_{0}\left(0, m_{A}^{2}, m_{h_{0}}{ }^{2}\right)-2 B_{0}\left(m_{Z}^{2}, m_{A}^{2}, m_{h_{0}}{ }^{2}\right)\right] \\
& \left.+m_{A}^{2}\left[-2 B_{0}\left(m_{Z}^{2}, m_{A}^{2}, m_{h_{0}}{ }^{2}\right)-2 B_{0}\left(0, m_{A}^{2}, m_{A}^{2}\right)+B_{0}\left(0, m_{A}^{2}, m_{h_{0}}{ }^{2}\right)\right]\right] \\
& +\frac{e^{2}\left(\sin ^{2} \theta_{W}-\cos ^{2} \theta_{W}\right)^{2}}{32 \cos ^{2} \theta_{W} \pi^{2} \sin ^{2} \theta_{W}} m_{H^{+}}{ }^{2}\left[1+B_{0}\left(0, m_{H^{+}}{ }^{2}, m_{H^{+}}{ }^{2}\right)\right] \\
& +\frac{e^{2}}{64 \cos ^{2} \theta_{W} \pi^{2} \sin ^{2} \theta_{W}} m_{H}^{2}\left[1+B_{0}\left(0, m_{H}^{2}, m_{H}^{2}\right)\right] \\
& +\frac{e^{2}}{64 \cos ^{2} \theta_{W} \pi^{2} \sin ^{2} \theta_{W}} m_{h_{0}}{ }^{2}\left[1+B_{0}\left(0, m_{h_{0}}{ }^{2}, m_{h_{0}}{ }^{2}\right)\right] \\
& +\frac{e^{2}}{64 \cos ^{2} \theta_{W} \pi^{2} \sin ^{2} \theta_{W}} m_{A}^{2}\left[1+B_{0}\left(0, m_{A}^{2}, m_{A}^{2}\right)\right] \\
& \begin{aligned}
\delta Z_{W} & =\frac{N_{C} e^{2}}{288 \pi^{2} m_{W}^{4} \sin ^{2} \theta_{W}}\left[2 m_{W}^{4}-6 m_{W}^{4} B_{0}\left(m_{W}^{2}, m_{b}^{2}, m_{t}^{2}\right)\right. \\
& +3 m_{t}^{4}\left[B_{0}\left(m_{W}^{2}, m_{b}^{2}, m_{t}^{2}\right)-B_{0}\left(m_{W}^{2}, m_{b}^{2}, m_{t}^{2}\right)\right] \\
& \left.+3 m_{W}^{2}\left(m_{t}^{4}+m_{t}^{2} m_{W}^{2}-2 m_{W}^{4}\right) G_{0}\left(m_{W}^{2}, m_{b}^{2}, m_{t}^{2}\right)\right]
\end{aligned}
\end{aligned}
$$




$$
\begin{aligned}
& +\frac{e^{2} \sin ^{2}(\beta-\alpha)}{576 m_{W}^{4} \pi^{2} \sin ^{2} \theta_{W}}\left[-2 m_{W}^{4}-3 m_{W}^{4} B_{0}\left(m_{W}^{2}, m_{H}^{2}, m_{H^{+}}{ }^{2}\right)\right. \\
& +3\left(m_{H}^{2}-m_{H^{+}}{ }^{2}\right)^{2}\left[-B_{0}\left(0, m_{H}^{2}, m_{H^{+}}{ }^{2}\right)+B_{0}\left(m_{W}^{2}, m_{H}^{2}, m_{H^{+}}{ }^{2}\right)\right] \\
& +\left[6 m_{H}^{2} m_{W}^{2} m_{H^{+}}{ }^{2}-3 m_{H}^{4} m_{W}^{2}-3 m_{H^{+}}{ }^{4} m_{W}^{2}\right. \\
& \left.\left.+6 m_{H}^{2} m_{W}^{4}+6 m_{H^{+}}{ }^{2} m_{W}^{4}-3 m_{W}^{6}\right] G_{0}\left(m_{W}^{2}, m_{H}^{2}, m_{H^{+}}{ }^{2}\right)\right] \\
& +\frac{e^{2} \cos ^{2}(\beta-\alpha)}{576 m_{W}^{4} \pi^{2} \sin ^{2} \theta_{W}}\left[-2 m_{W}^{4}-3 m_{W}^{4} B_{0}\left(m_{W}^{2}, m_{H^{+}}{ }^{2}, m_{h_{0}}{ }^{2}\right)\right. \\
& +3\left({m_{h_{0}}}^{2}-m_{H^{+}}{ }^{2}\right)^{2}\left[B_{0}\left(m_{W}^{2}, m_{H^{+}}{ }^{2}, m_{h_{0}}{ }^{2}\right)-B_{0}\left(0, m_{H^{+}}{ }^{2}, m_{h_{0}}{ }^{2}\right)\right] \\
& +\left[6 m_{h_{0}}{ }^{2} m_{W}^{2} m_{H^{+}}{ }^{2}-3 m_{h_{0}}{ }^{4} m_{W}^{2}-3 m_{H^{+}}{ }^{4} m_{W}^{2}\right. \\
& \left.\left.+6 m_{h_{0}}{ }^{2} m_{W}^{4}+6 m_{H^{+}}{ }^{2} m_{W}^{4}-3 m_{W}^{6}\right] G_{0}\left(m_{W}^{2}, m_{H^{+}}{ }^{2}, m_{h_{0}}{ }^{2}\right)\right] \\
& +\frac{e^{2}}{576 m_{W}^{4} \pi^{2} \sin ^{2} \theta_{W}}\left[-2 m_{W}^{4}-3 m_{W}^{4} B_{0}\left(m_{W}^{2}, m_{A}^{2}, m_{H^{+}}{ }^{2}\right)\right. \\
& +3\left(m_{A}^{2}-m_{H^{+}}{ }^{2}\right)^{2}\left[-B_{0}\left(0, m_{A}^{2}, m_{H^{+}}{ }^{2}\right)+B_{0}\left(m_{W}^{2}, m_{A}^{2}, m_{H^{+}}{ }^{2}\right)\right] \\
& +\left[6 m_{A}^{2} m_{W}^{2} m_{H^{+}}{ }^{2}-3 m_{A}^{4} m_{W}^{2}-3 m_{H^{+}}{ }^{4} m_{W}^{2}\right. \\
& \left.\left.+6 m_{A}^{2} m_{W}^{4}+6 m_{H^{+}}{ }^{2} m_{W}^{4}-3 m_{W}^{6}\right] G_{0}\left(m_{W}^{2}, m_{A}^{2}, m_{H^{+}}{ }^{2}\right)\right] \\
& +\frac{e^{2} \cos ^{2}(\beta-\alpha)}{576 m_{W}^{4} \pi^{2} \sin ^{2} \theta_{W}}\left[-2 m_{W}^{2}-3 m_{W}^{4} B_{0}\left(0, m_{H}^{2}, m_{W}^{2}\right)\right. \\
& +3 m_{H}^{4}\left[B_{0}\left(m_{W}^{2}, m_{H}^{2}, m_{W}^{2}\right)-B_{0}\left(0, m_{H}^{2}, m_{W}^{2}\right)\right] \\
& +6 m_{H}^{2} m_{W}^{2}\left[B_{0}\left(0, m_{H}^{2}, m_{W}^{2}\right)-B_{0}\left(m_{W}^{2}, m_{H}^{2}, m_{W}^{2}\right)\right] \\
& \left.+\left(12 m_{H}^{2} m_{W}^{4}-3 m_{H}^{4} m_{W}^{2}\right) G_{0}\left(m_{W}^{2}, m_{H}^{2}, m_{W}^{2}\right)\right] \\
& +\frac{e^{2} \sin ^{2}(\beta-\alpha)}{576 m_{W}^{4} \pi^{2} \sin ^{2} \theta_{W}}\left[-2 m_{W}^{2}-3 m_{W}^{4} B_{0}\left(0, m_{h_{0}}{ }^{2}, m_{W}^{2}\right)\right. \\
& +3 m_{h_{0}}{ }^{4}\left[B_{0}\left(m_{W}^{2}, m_{h_{0}}{ }^{2}, m_{W}^{2}\right)-B_{0}\left(0, m_{h_{0}}{ }^{2}, m_{W}^{2}\right)\right] \\
& +6 m_{h_{0}}{ }^{2} m_{W}^{2}\left[B_{0}\left(0, m_{h_{0}}{ }^{2}, m_{W}^{2}\right)-B_{0}\left(m_{W}^{2}, m_{h_{0}}{ }^{2}, m_{W}^{2}\right)\right] \\
& \left.+\left(12 m_{h_{0}}{ }^{2} m_{W}^{4}-3 m_{h_{0}}{ }^{4} m_{W}{ }_{W}\right) G_{0}\left(m_{W}^{2}, m_{h_{0}}{ }^{2}, m_{W}{ }_{W}\right)\right]
\end{aligned}
$$

$$
\begin{aligned}
\delta Z_{H^{ \pm} W}{ }^{1 / 2} & =\frac{N_{C} e^{2} m_{t}^{2} \cot \beta}{32 m_{H^{+}}{ }^{2} m_{W}^{3} \pi^{2} \sin ^{2} \theta_{W}}\left[m_{t}^{2}\left(B_{0}\left(0, m_{b}^{2}, m_{t}^{2}\right)-B_{0}\left(m_{H^{+}}{ }^{2}, m_{b}^{2}, m_{t}^{2}\right)\right)\right. \\
& \left.+m_{H^{+}}{ }^{2} B_{0}\left(m_{H^{+}}{ }^{2}, m_{b}^{2}, m_{t}^{2}\right)\right] \\
& -\frac{e^{2} \sin (\beta-\alpha)\left(m_{H^{+}}{ }^{2}-m_{H}^{2}\right)}{64 m_{W}^{2} m_{H^{+}}{ }^{2} \pi^{2} \sin ^{2} \theta_{W} \cos \theta_{W}}\left[2 \cos \theta_{W} m_{W} \cos (\alpha-\beta)-m_{Z} \cos 2 \beta \cos (\alpha+\beta)\right] \\
& \times\left[B_{0}\left(m_{H^{+}}{ }^{2}, m_{H}{ }^{2}, m_{H^{+}}{ }^{2}\right)-B_{0}\left(0, m_{H}{ }^{2}, m_{H^{+}}{ }^{2}\right)\right]
\end{aligned}
$$




$$
\begin{aligned}
& +\frac{e^{2} \sin (\beta+\alpha)\left(m_{h_{0}}{ }^{2}-m_{H^{+}}{ }^{2}\right)}{64 m_{W}^{2} m_{H^{+}}{ }^{2} \pi^{2} \sin ^{2} \theta_{W} \cos \theta_{W}}\left[2 \cos \theta_{W} m_{W} \sin (\alpha-\beta)-m_{Z} \cos 2 \beta \sin (\alpha+\beta)\right] \\
& \times\left[B_{0}\left(m_{H^{+}}{ }^{2}, m_{H^{+}}{ }^{2}, m_{h_{0}}{ }^{2}\right)-B_{0}\left(0, m_{H^{+}}{ }^{2}, m_{h_{0}}{ }^{2}\right)\right] \\
& +\frac{e^{2} \sin 2 \beta\left(m_{H}^{2}-m_{W}^{2}\right)}{64 m_{W}^{2} m_{H^{+}}{ }^{2} \pi^{2} \sin ^{2} \theta_{W} \cos \theta_{W}}\left[\cos \theta_{W} m_{W} \sin (\alpha-\beta)+m_{Z} \cos (\beta+\alpha) \sin (2 \beta)\right] \\
& \times\left[-B_{0}\left(m_{H^{+}}{ }^{2}, m_{H}^{2}, m_{W}^{2}\right)+B_{0}\left(0, m_{H}^{2}, m_{W}^{2}\right)\right] \\
& +\frac{e^{2} \sin (\alpha-\beta)\left(m_{h_{0}}{ }^{2}-m_{W}^{2}\right)}{64 m_{W}^{2} m_{H^{+}}{ }^{2} \pi^{2} \sin ^{2} \theta_{W} \cos \theta_{W}}\left[-\cos \theta_{W} m_{W} \cos (\alpha-\beta)+m_{Z} \sin (\beta+\alpha) \sin (2 \beta)\right] \\
& \times\left[-B_{0}\left(m_{H^{+}}{ }^{2}, m_{h_{0}}{ }^{2}, m_{W}^{2}\right)+B_{0}\left(0, m_{h_{0}}{ }^{2}, m_{W}^{2}\right)\right] \\
& +\frac{e^{2} \sin (\alpha-\beta) \cos (\alpha-\beta)}{64 m_{W} m_{H^{+}}{ }^{2} \pi^{2} \sin ^{2} \theta_{W}}\left[m_{H}^{2}\left[B_{0}\left(0, m_{H}^{2}, m_{W}^{2}\right)-B_{0}\left(m_{H^{+}}{ }^{2}, m_{H}^{2}, m_{W}^{2}\right)\right]\right. \\
& \left.-3 m_{H^{+}}{ }^{2} B_{0}\left(m_{H^{+}}{ }^{2}, m_{H}^{2}, m_{W}^{2}\right)\right] \\
& +\frac{e^{2} \sin ^{2}(\alpha-\beta) \cos ^{2}(\alpha-\beta)}{64 m_{W} m_{H^{+}}{ }^{2} \pi^{2} \sin ^{2} \theta_{W}}\left[m_{h_{0}}{ }^{2}\left[-B_{0}\left(0, m_{h_{0}}{ }^{2}, m_{W}^{2}\right)+B_{0}\left(m_{H^{+}}{ }^{2}, m_{h_{0}}{ }^{2}, m_{W}^{2}\right)\right]\right. \\
& \left.+3 m_{H^{+}}{ }^{2} B_{0}\left(m_{H^{+}}{ }^{2}, m_{h_{0}}{ }^{2}, m_{W}^{2}\right)\right]
\end{aligned}
$$

$$
\begin{aligned}
\delta Z_{H^{ \pm}} & =\frac{N_{C} e^{2} m_{t}^{2} \cot (\beta)^{2}}{32 m_{W}^{2} \pi^{2} \sin ^{2} \theta_{W}}\left[-B_{0}\left(m_{H^{+}}{ }^{2}, m_{b}^{2}, m_{t}^{2}\right)+\left(m_{t}^{2}-m_{H^{+}}{ }^{2}\right) G_{0}\left(m_{H^{+}}{ }^{2}, m_{W}^{2}, m_{t}^{2}\right)\right] \\
& +\frac{e^{2}\left(\sin ^{2} \theta_{W}-\cos ^{2} \theta_{W}\right)^{2} m_{H^{+}}{ }^{2}}{16 \pi^{2} \sin ^{2} \theta_{W} \cos ^{2} \theta_{W}} G_{0}\left(m_{H^{+}}{ }^{2}, m_{H^{+}}{ }^{2}, m_{Z}^{2}\right) \\
& +\frac{e^{2} \sin (\alpha-\beta)^{2}\left(m_{H^{+}}{ }^{2}+m_{H}^{2}\right)}{32 \pi^{2} \sin ^{2} \theta_{W}} G_{0}\left(m_{H^{+}}{ }^{2}, m_{H}^{2}, m_{W}^{2}\right) \\
& +\frac{e^{2} \cos ^{2}(\alpha-\beta)\left(m_{H_{0}}{ }^{2}+m_{H^{+}}{ }^{2}\right)}{32 \pi^{2} \sin ^{2} \theta_{W}} G_{0}\left(m_{H^{+}}{ }^{2}, m_{H_{0}}{ }^{2}, m_{W}^{2}\right) \\
& +\frac{e^{2}\left(m_{H^{+}}{ }^{2}+m_{A}^{2}\right)}{32 \pi^{2} \sin ^{2} \theta_{W}} G_{0}\left(m_{H^{+}}{ }^{2}, m_{A}^{2}, m_{W}^{2}\right)
\end{aligned}
$$

$$
\begin{aligned}
\delta Z_{h_{0}} & =\frac{N_{C} e^{2} m_{t}^{2}}{32 m_{W}^{2} \pi^{2}} \cos ^{2} \alpha \csc ^{2} \beta\left[-B_{0}\left(m_{h_{0}}{ }^{2}, m_{t}^{2}, m_{t}^{2}\right)+\left(4 m_{t}^{2}-m_{h_{0}}{ }^{2}\right) G_{0}\left(m_{h_{0}}{ }^{2}, m_{t}^{2}, m_{t}^{2}\right)\right] \\
& +\frac{e^{2} \cos ^{2}(\alpha-\beta)\left(m_{H^{+}}{ }^{2}+m_{h_{0}}{ }^{2}\right)}{16 \pi^{2} \sin ^{2} \theta_{W}} G_{0}\left(m_{h_{0}}{ }^{2}, m_{H^{+}}{ }^{2}, m_{W}^{2}\right) \\
& +\frac{e^{2} \sin ^{2}(\alpha-\beta) m_{h_{0}}{ }^{2}}{16 \pi^{2} \sin ^{2} \theta_{W}} G_{0}\left(m_{h_{0}}{ }^{2}, m_{W}^{2}, m_{W}^{2}\right) \\
& +\frac{e^{2} \sin ^{2}(\alpha-\beta) m_{h_{0}}{ }^{2}}{32 \pi^{2} \sin ^{2} \theta_{W} \cos ^{2} \theta_{W}} G_{0}\left(m_{h_{0}}{ }^{2}, m_{Z}^{2}, m_{Z}^{2}\right) \\
& +\frac{e^{2} \cos ^{2}(\alpha-\beta)\left(m_{A}^{2}+m_{h_{0}}{ }^{2}\right)}{32 \pi^{2} \sin ^{2} \theta_{W} \cos ^{2} \theta_{W}} G_{0}\left(m_{h_{0}}{ }^{2}, m_{A}^{2}, m_{Z}^{2}\right)
\end{aligned}
$$


Here and below, $B_{0}, C_{0}, C_{i}$ and $C_{i j}$ is the two-point and three-point scalar integrals, definitions for which can be found in Ref. [12] and $G_{0}$ is the derivative of $B_{0}$ which is expressed as

$$
G_{0}\left(M^{2}, m_{1}^{2}, m_{2}^{2}\right)=\left.\frac{\partial B_{0}\left(k^{2}, m_{1}^{2}, m_{2}^{2}\right)}{\partial k^{2}}\right|_{k^{2}=M^{2}}
$$

\section{APPENDIX-B: SELF-ENERGY CORRECTIONS}

$$
\begin{aligned}
& \delta M_{T}^{\text {self }}=\frac{N_{c} e^{4} m_{W} \sin (\beta-\alpha)}{288 \sqrt{2} \pi^{2} \hat{s}\left(-m_{W}^{2}+\hat{s}\right)^{2} \sin \theta_{w}^{4}}\left[6 \hat{s} m_{t}^{2}-2 \hat{s}^{2}+3 m_{t}^{2}\left(m_{t}^{2}-2 \hat{s}\right)\right. \\
& \left.\times B_{0}\left(0, m_{b}^{2}, m_{t}^{2}\right)+3\left(-m_{t}^{4}-\hat{s} m_{t}^{2}+2 \hat{s}^{2}\right) B_{0}\left(\hat{s}, m_{b}^{2}, m_{t}^{2}\right)\right] \\
& \delta M_{H}^{\text {self }}=-\frac{e^{4} m_{W} \sin ^{3}(\beta-\alpha)}{64 \sqrt{2} \pi^{2}\left(m_{W}^{2}-\hat{s}\right)^{2} \sin ^{4} \theta_{W}}\left[\frac{2}{9}\left(3 m_{H}^{2}-6 m_{H^{+}}^{2}-\hat{s}\right)+\frac{2 m_{H^{+}}^{2}}{3} B_{0}\left(0, m_{H^{+}}^{2}, m_{H^{+}}^{2}\right)\right. \\
& +\frac{\left(m_{H^{+}}^{2}-m_{H}^{2}\right)\left(m_{H^{+}}^{2}-m_{H}^{2}-\hat{s}\right)}{3 \hat{s}} B_{0}\left(0, m_{H}^{2}, m_{H^{+}}^{2}\right) \\
& \left.+\frac{\left[\left(m_{H^{+}}-m_{H}\right)^{2}-\hat{s}\right]\left[\hat{s}-\left(m_{H^{+}}+m_{H}\right)^{2}\right]}{3 \hat{s}} B_{0}\left(\hat{s}, m_{H}, m_{H^{+}}^{2}\right)\right] \\
& -\frac{e^{4} m_{W} \cos ^{2}(\beta-\alpha) \sin (\beta-\alpha)}{64 \sqrt{2} \pi^{2}\left(m_{W}^{2}-\hat{s}\right)^{2} \sin ^{4} \theta_{W}}\left[\frac{2}{9}\left(3 m_{h_{0}}^{2}+6 m_{H^{+}}^{2}-\hat{s}\right)+\frac{2 m_{H^{+}}^{2}}{3} B_{0}\left(0, m_{H^{+}}^{2}, m_{H^{+}}^{2}\right)\right. \\
& +\frac{\left(m_{H^{+}}^{2}-m_{h_{0}}^{2}\right)\left(m_{H^{+}}^{2}-m_{h_{0}}^{2}-\hat{s}\right)}{3 \hat{s}} B_{0}\left(0, m_{H^{+}}^{2}, m_{h_{0}}^{2}\right) \\
& \left.+\frac{\left[\left(m_{H^{+}}-m_{h_{0}}\right)^{2}-\hat{s}\right]\left[\hat{s}-\left(m_{H^{+}}+m_{h_{0}}\right)^{2}\right]}{3 \hat{s}} B_{0}\left(\hat{s}, m_{H^{+}}^{2}, m_{h_{0}}^{2}\right)\right] \\
& +\frac{e^{4} m_{W} \sin (\beta-\alpha)}{64 \sqrt{2} \pi^{2}\left(m_{W}^{2}-\hat{s}\right)^{2} \sin ^{4} \theta_{W}}\left[\frac{2}{9}\left(6 m_{A}^{2}+3 m_{H^{+}}^{2}-\hat{s}\right)+\frac{2 m_{A}^{2}}{3} B_{0}\left(0, m_{A}^{2}, m_{A}^{2}\right)\right. \\
& +\frac{\left(m_{H^{+}}^{2}-m_{A}^{2}\right)\left(m_{H^{+}}^{2}-m_{A}^{2}-\hat{s}\right)}{3 \hat{s}} B_{0}\left(0, m_{A}^{2}, m_{H^{+}}^{2}\right) \\
& \left.+\frac{\left[\left(m_{H^{+}}-m_{A}\right)^{2}-\hat{s}\right]\left[\hat{s}-\left(m_{H^{+}}+m_{A}\right)^{2}\right]}{3 \hat{s}} B_{0}\left(\hat{s}, m_{A}, m_{H^{+}}^{2}\right)\right] \\
& -\frac{e^{4} m_{W} \sin (\beta-\alpha) \cos ^{2}(\beta-\alpha)}{64 \sqrt{2} \pi^{2}\left(m_{W}^{2}-\hat{s}\right)^{2} \sin ^{4} \theta_{W}}\left[\frac{2}{9}\left(6 m_{H}^{2}-\hat{s}\right)+\frac{2 m_{H}^{2}}{3} B_{0}\left(0, m_{H}^{2}, m_{H}^{2}\right)\right. \\
& +\left(\frac{\left(m_{H}^{2}-m_{W}^{2}\right)\left(m_{H}^{2}-m_{W}^{2}-\hat{s}\right)}{3 \hat{s}}-\frac{m_{W}^{2}}{3}\right) B_{0}\left(0, m_{H}^{2}, m_{W}^{2}\right) \\
& \left.+\left(\frac{\left[\left(m_{H}-m_{W}\right)^{2}-\hat{s}\right]\left[\hat{s}-\left(m_{H}+m_{W}\right)^{2}\right]}{3 \hat{s}}-\frac{2 m_{W}^{2}}{3}\right) B_{0}\left(\hat{s}, m_{H}^{2}, m_{W}^{2}\right)\right] \\
& -\frac{e^{4} m_{W} \sin (\beta-\alpha)^{3}}{64 \sqrt{2} \pi^{2}\left(m_{W}^{2}-\hat{s}\right)^{2} \sin ^{4} \theta_{W}}\left[\frac{2}{9}\left(6 m_{h_{0}}^{2}-\hat{s}\right)+\frac{2 m_{h_{0}}^{2}}{3} B_{0}\left(0, m_{h_{0}}^{2}, m_{h_{0}}^{2}\right)\right.
\end{aligned}
$$




$$
\begin{aligned}
& +\left(\frac{\left(m_{h_{0}}^{2}-m_{W}^{2}\right)\left(m_{h_{0}}^{2}-m_{W}^{2}-\hat{s}\right)}{3 \hat{s}}-\frac{m_{W}^{2}}{3}\right) B_{0}\left(0, m_{h_{0}}^{2}, m_{W}^{2}\right) \\
& \left.+\left(\frac{\left[\left(m_{h_{0}}-m_{W}\right)^{2}-\hat{s}\right]\left[\hat{s}-\left(m_{h_{0}}+m_{W}\right)^{2}\right]}{3 \hat{s}}-\frac{2 m_{W}^{2}}{3}\right) B_{0}\left(\hat{s}, m_{h_{0}}^{2}, m_{W}^{2}\right)\right] \\
& +\frac{e^{4} m_{W} \sin (\beta-\alpha) m_{H}^{2}}{64 \sqrt{2} \pi^{2}\left(m_{W}^{2}-\hat{s}\right)^{2} \sin ^{4} \theta_{W}}\left[1+B_{0}\left(0, m_{H}^{2}, m_{H}^{2}\right)\right] \\
& +\frac{e^{4} m_{W} \sin (\beta-\alpha) m_{H^{+}}^{2}}{64 \sqrt{2} \pi^{2}\left(m_{W}^{2}-\hat{s}\right)^{2} \sin ^{4} \theta_{W}}\left[1+B_{0}\left(0, m_{H^{+}}^{2}, m_{H^{+}}^{2}\right)\right] \\
& +\frac{e^{4} m_{W} \sin (\beta-\alpha) m_{h_{0}}^{2}}{64 \sqrt{2} \pi^{2}\left(m_{W}^{2}-\hat{s}\right)^{2} \sin ^{4} \theta_{W}}\left[1+B_{0}\left(0, m_{h_{0}}^{2}, m_{h_{0}}^{2}\right)\right] \\
& +\frac{e^{4} m_{W} \sin (\beta-\alpha) m_{A}^{2}}{64 \sqrt{2} \pi^{2}\left(m_{W}^{2}-\hat{s}\right)^{2} \sin ^{4} \theta_{W}}\left[1+B_{0}\left(0, m_{A}^{2}, m_{A}^{2}\right)\right]
\end{aligned}
$$

\section{APPENDIX-C: THE VERTEX FORM FACTORS}

$$
\begin{aligned}
f_{1}^{\text {vertex }}= & \frac{-N_{c} e^{4} m_{t}^{2}}{32 \sqrt{2} m_{W} \pi^{2}\left(m_{W}{ }^{2}-\hat{s}\right) \sin \theta_{w}{ }^{4}}\left[-2 B_{0}\left(\hat{s}, m_{b}^{2}, m_{t}^{2}\right)\right. \\
& +\left(-2 m_{t}^{2}-\hat{t}\right) C_{0}\left(m_{h_{0}}^{2}, m_{W}^{2}, \hat{s}, m_{t}^{2}, m_{t}^{2}, m_{b}^{2}\right) \\
& +\left(-2 m_{W}^{2}-\hat{t}\right) C_{1}\left(m_{W}^{2}, \hat{s}, m_{h_{0}}^{2}, m_{t}^{2}, m_{b}^{2}, m_{t}^{2}\right) \\
& +\left(-m_{h_{0}}^{2}-2 \hat{t}\right) C_{2}\left(m_{W}^{2}, \hat{s}, m_{h_{0}}^{2}, m_{t}^{2}, m_{b}^{2}, m_{t}^{2}\right) \\
& \left.\quad+4 C_{00}\left(m_{W}^{2}, \hat{s}, m_{h_{0}}^{2}, m_{t}^{2}, m_{b}^{2}, m_{t}^{2}\right)\right] \\
f_{2}^{\text {vertex }}= & \frac{-N_{c} e^{4} m_{t}^{2}}{16 \sqrt{2} m_{W} \pi^{2}\left(m_{W}{ }^{2}-\hat{s}\right) \sin \theta_{w}{ }^{4}}\left[-C_{0}\left(m_{h_{0}}^{2}, m_{W}^{2}, \hat{s}, m_{t}^{2}, m_{t}^{2}, m_{b}^{2}\right)\right. \\
- & C_{1}\left(m_{W}^{2}, \hat{s}, m_{h_{0}}^{2}, m_{t}^{2}, m_{b}^{2}, m_{t}^{2}\right) \\
- & 3 C_{2}\left(m_{W}^{2}, \hat{s}, m_{h_{0}}^{2}, m_{t}^{2}, m_{b}^{2}, m_{t}^{2}\right) \\
- & 2 C_{12}\left(m_{W}^{2}, \hat{s}, m_{h_{0}}^{2}, m_{t}^{2}, m_{b}^{2}, m_{t}^{2}\right) \\
- & \left.2 C_{22}\left(m_{W}^{2}, \hat{s}, m_{h_{0}}^{2}, m_{t}^{2}, m_{b}^{2}, m_{t}^{2}\right)\right] \\
f_{3}^{\text {vertex }}= & \frac{-N_{c} e^{4} m_{t}^{2}}{16 \sqrt{2} m_{W} \pi^{2}\left(m_{W}{ }^{2}-\hat{s}\right) \sin \theta_{w}{ }^{4}}\left[-C_{2}\left(m_{W}^{2}, \hat{s}, m_{h_{0}}^{2}, m_{t}^{2}, m_{b}^{2}, m_{t}^{2}\right)\right. \\
- & 2 C_{12}\left(m_{W}^{2}, \hat{s}, m_{h_{0}}^{2}, m_{t}^{2}, m_{b}^{2}, m_{t}^{2}\right) \\
- & \left.2 C_{22}\left(m_{W}^{2}, \hat{s}, m_{h_{0}}^{2}, m_{t}^{2}, m_{b}^{2}, m_{t}^{2}\right)\right] .
\end{aligned}
$$


[1] P. McNamara, ICHEP '98, Vancouver, July 1998.

[2] C. Quigg, FERMILAB-CONF-98/059-T, hep-ph/9802320.

[3] A. Stange, W. Marciano, and S. Willenbrock, Phys. Rev. D49, 1354 (1994); ibid. D50,4491 (1994);

S. Kim, S. Kuhlman, and W.Yao, CDF-ANAL-EXOTIC-PUBLIC-3904, Oct. 96;

W.Yao, FERMILAB-CONF-96-383-E, Jun. 96;

J. Womersley, D0 Note 3227, Apr. 97;

S. Parke, FERMILAB-CONF-97/335-T.

[4] T. Han and S. Willenbrock, Phys. Lett. B273, 167 (1990);

J. Ohnemus and W.J. Stirling, Phys. Rev. D47, 2722 (1993);

H. Baer, B. Bailay, and J.F. Owens, Phys. Rev. D47, 2730 (1993);

S. Smith and S. Willenbrock, Phys. Rev. D54, 6696 (1996).

[5] H.E. Haber and G.L. Kane, Phys. Rep. 117, 75(1985);

J.F. Gunion and H.E. Haber, Nucl. Phys. B 272, 1 (1986).

[6] J. Gunion and A. Turski, Phys. Rev. D39 , 2701 (1989) and D40 2333 (1990);

J.R. Espinosa and M. Quiros, Phys. Lett. B266, 389 (1991);

M. Carena, M. Quiros, and C.E.M. Wagner, Nucl. Phys. B461, 407 (1996).

[7] C.S. Li and S.H Zhu, to appear in Phys. Lett. B, hep-ph/9801390.

[8] S. Sirlin, Phys. Rev. D22, 971 (1980); W. J. Marciano and A. Sirlin, ibid. 22 , 2695 (1980);31, 213(E) (1985); A. Sirlin and W.J. Marciano, Nucl. Phys. B189, 442 (1981); K.I. Aoki et.al., Prog. Theor. Phys. Suppl. 73, 1 (1982).

[9] A. Mendez and A. Pomarol, Phys. Lett. B279, 98 (1992).

[10] H.L. Lai, J. Botts, J. Huston, J.R. Mofin, J.F. Owens, J.W. Qiu, W.K. Tung, and H. Weerts, Phys. Rev. D51, 4763 (1995). 
[11] M. Spiral, CERN-TH/97-68 (hep-ph/9705337).

[12] G. Passarino and M. Veltman, Nucl.Phys. B33, 151 (1979); A. Denner, Fortschr. Phys. 41 (1993)4. 


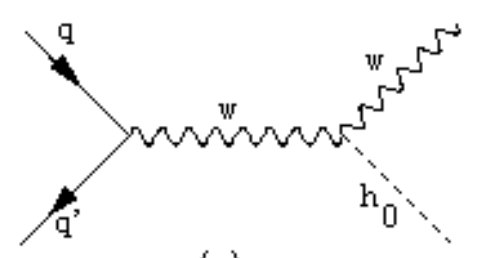

(a)
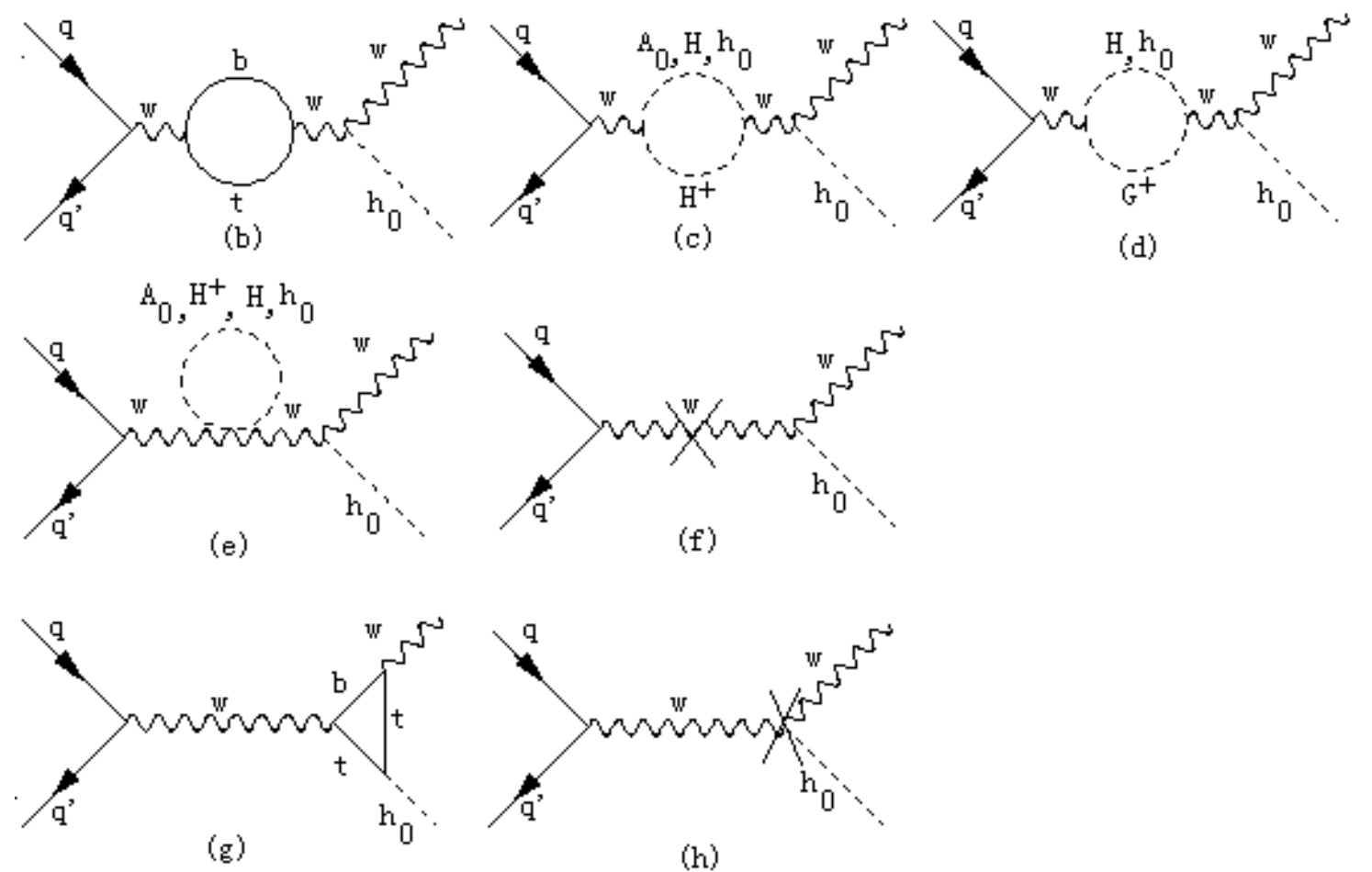

(h)

FIG. 1.

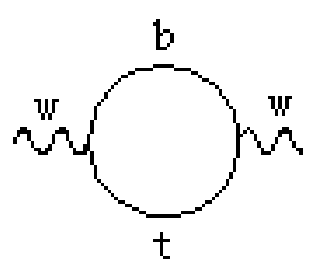

(a)

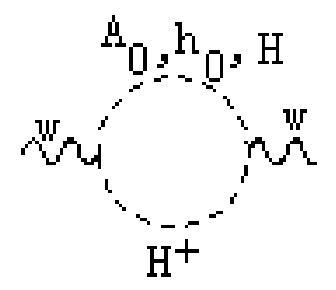

(b)

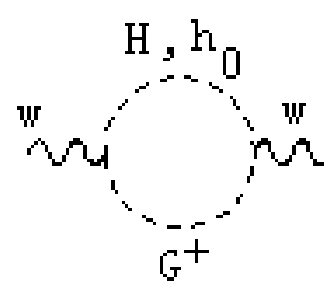

(c)

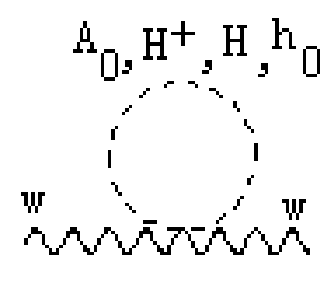

(d)

FIG. 2. 


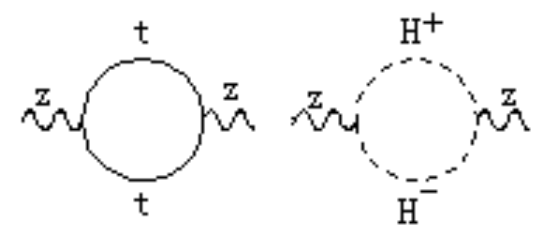

(a)

(b)

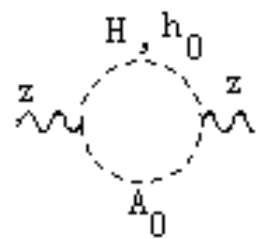

(c)

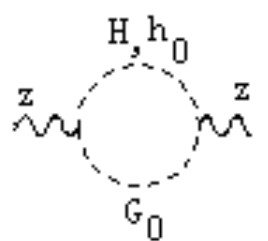

(d)

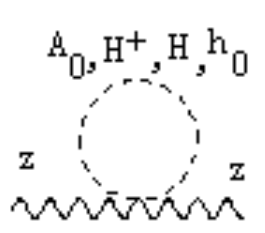

(e)

FIG. 3.

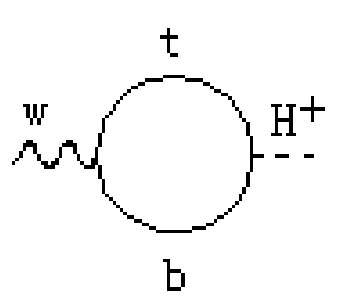

(a)

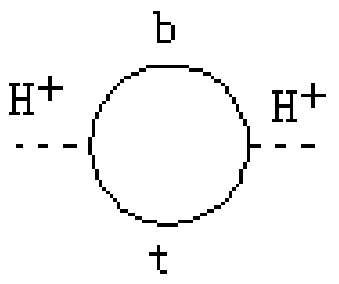

(d)

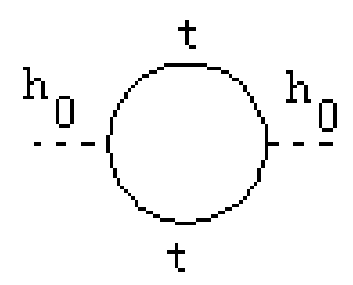

(g)

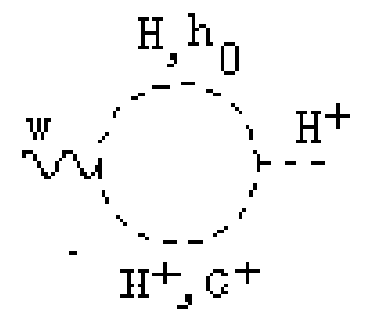

(b)

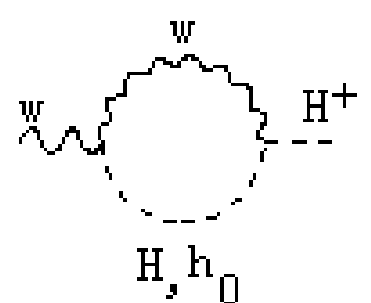

(c)

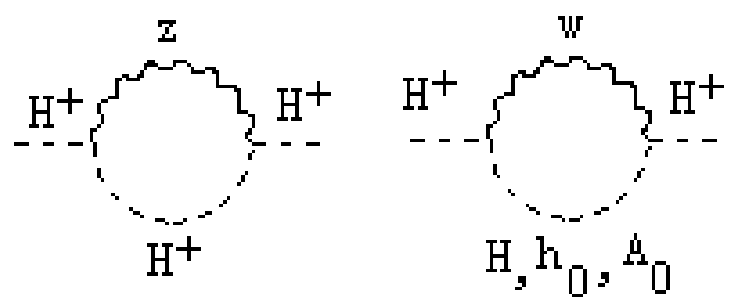

(e)

(f)

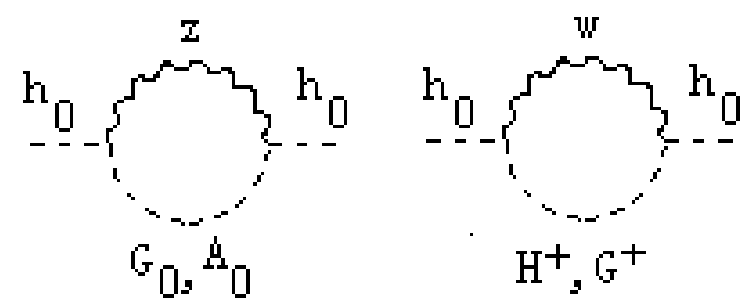

(h)

(i)

FIG. 4. 


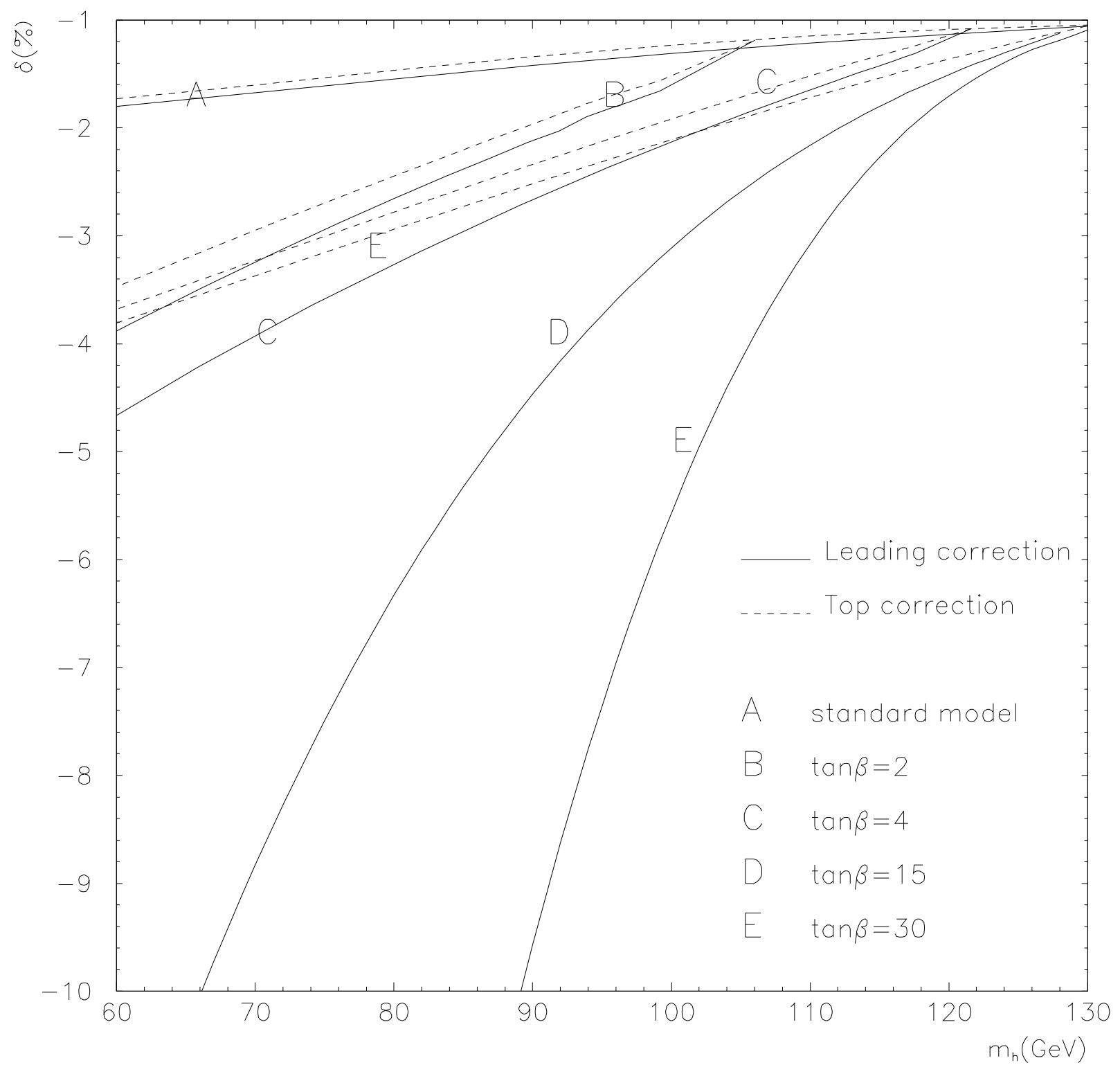

FIG. 5. Relative corrections $\delta \sigma / \sigma_{0}$ as a function of the Higgs boson mass of the process $q \bar{q}^{\prime} \rightarrow W h_{0}$ with $\sqrt{s}=2 T e V$ at Tevatron. 


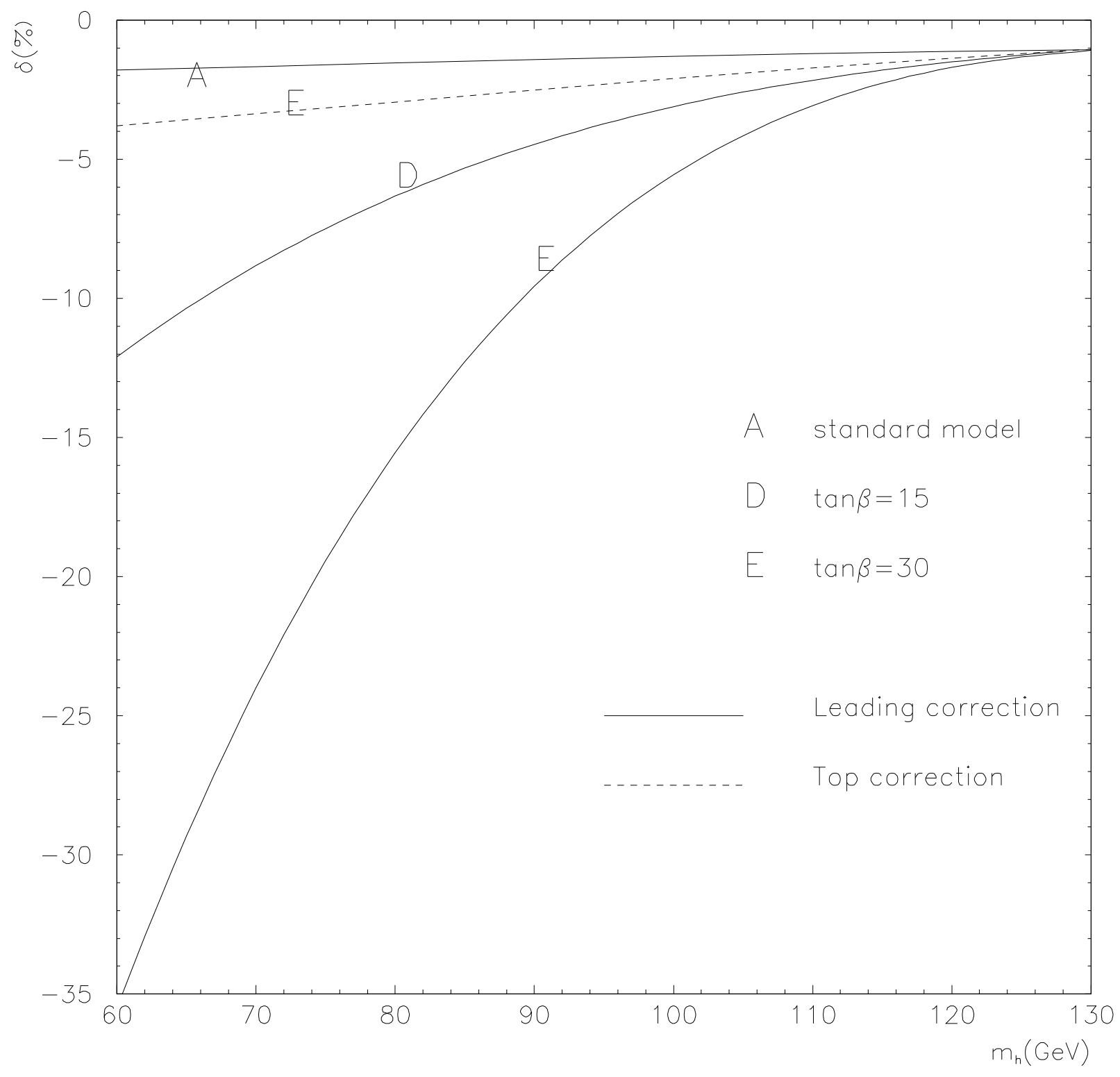

FIG. 6. Relative corrections $\delta \sigma / \sigma_{0}$ as a function of the Higgs boson mass of the process $q \bar{q}^{\prime} \rightarrow W h_{0}$ with $\sqrt{s}=2 T e V$ at Tevatron. 